\title{
Left ventricular assist device implantation with axillary- axillary outflow graft
}

\author{
Dominique L. Tucker, BS, ${ }^{\mathrm{a}}$ John Perry, MD, ${ }^{\mathrm{b}}$ Ashley Bock, MD, ${ }^{\mathrm{c}}$ Aaron Douglas, DO, ${ }^{\mathrm{d}}$ \\ Chonyang Albert, MD, ${ }^{\mathrm{c}}$ Lee Kirksey, MD, MBA, ${ }^{\mathrm{e}}$ and Michael Zhen-Yu Tong, MD, ${ }^{\mathrm{b}}$ Cleveland, Ohio
}

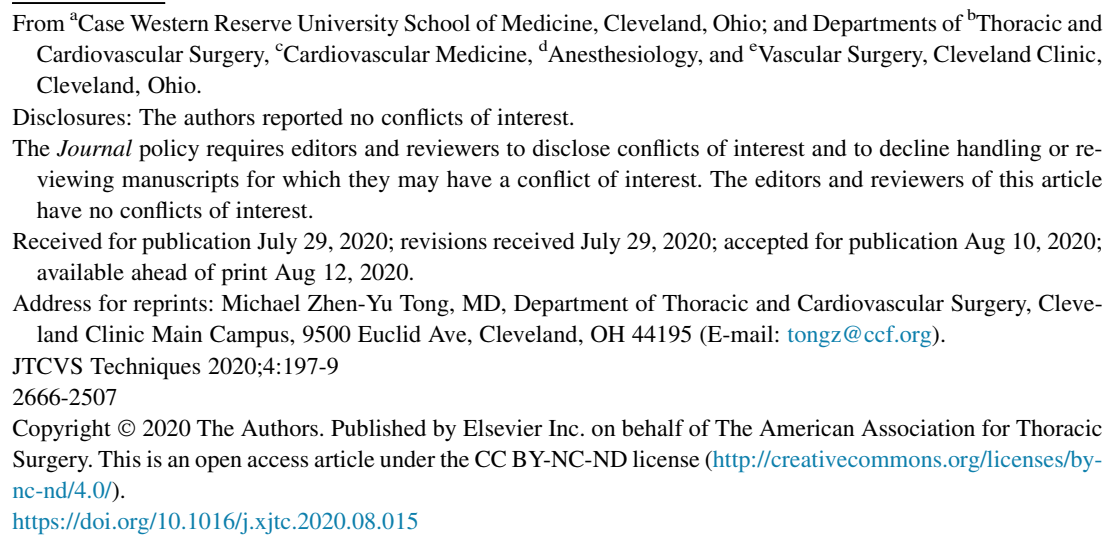

Left ventricular assist devices (LVADs) are used for patients with end-stage heart disease either as a bridge to transplant or as destination therapy. Although the most common configuration is LV apex to ascending aorta through a sternotomy, patient anatomy and clinical circumstances may necessitate alternative configurations. ${ }^{1}$ Herein, we describe a novel alternative approach to augment LVAD flow with a left axillary-right axillary arterial bypass graft (LARAAB).

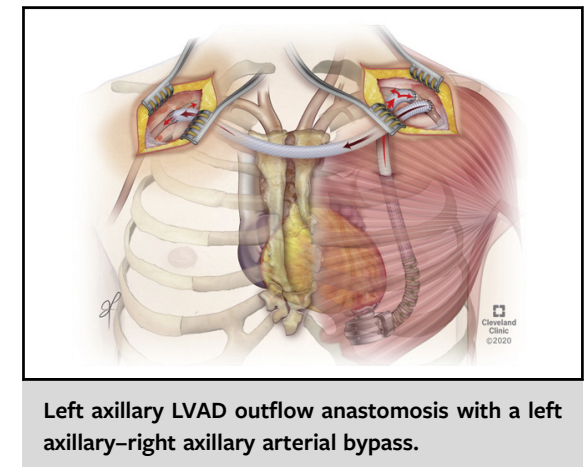

CENTRAL MESSAGE

We describe a novel alternative approach to augment left ventricular assist device outflow in the setting of a porcelain ascending aorta, hostile chest wall, and a small caliber axillary artery.

See Commentaries on pages 200, 202, and 204

\section{CASE SUMMARY}

A 60-year-old woman with a history of ischemic cardiomyopathy presented with cardiogenic shock. She had numerous comorbidities, including morbid obesity (body mass index of 50), type 2 diabetes mellitus, current smoking, and multiple rounds of chest irradiation with lumpectomy for breast cancer with multiple reconstructions. She has also had a previous coronary artery bypass graft complicated by mediastinitis requiring pectoral sternal flaps and her ascending aorta was heavily calcified (Figure 1). We performed LVAD with HVAD (Medtronic, Fridley, Minn) placement through a left thoracotomy with outflow to the left axillary artery. Preoperatively, she had no discrepancy in bilateral arm pressures. The left subclavian artery measured $7 \mathrm{~mm}$ in diameter and demonstrated no evidence of atherosclerotic disease on computed tomography angiography and ultrasound.

Intraoperatively, blood pressure was monitored via arterial lines placed in bilateral radial arteries and the right common femoral artery. Cardiopulmonary bypass (CPB) was cannulated via the left groin. The centrifugal LVAD was implanted in the LV apex through the sixth intercostal space.
Dense adhesions of the lungs were lysed to permit passage of the outflow to the left axillary artery through the second intercostal space; however, dense fibrosis was prohibitive for access to the descending aorta.

Upon weaning from CPB, the mean arterial pressure (MAP) gradients between the left and right axillary arteries were $>40 \mathrm{~mm} \mathrm{Hg}$. The axillary anastomosis was assessed by Doppler examination and was free of stenosis. An axillary band was placed distal to the anastomosis to reduce arm hyperperfusion. The arm-to-arm MAP gradient subsequently decreased to $<15 \mathrm{~mm} \mathrm{Hg}$. Increases in LVAD revolutions per minute increased the left arm pressure, but the right arm and femoral pressures and flows peaked at approximately $2 \mathrm{~L}$ with partial decompression of the LV. With moderate to high doses of inotropic support, the patient was successfully weaned from CPB. We left the operating room with LVAD flow of $2.2 \mathrm{~L} / \mathrm{min}$ on $3040 \mathrm{rpm}$ with a MAP of 90 and $75 \mathrm{~mm} \mathrm{Hg}$ measured at the left and right arm/femoral artery, respectively. The overall cardiac index 


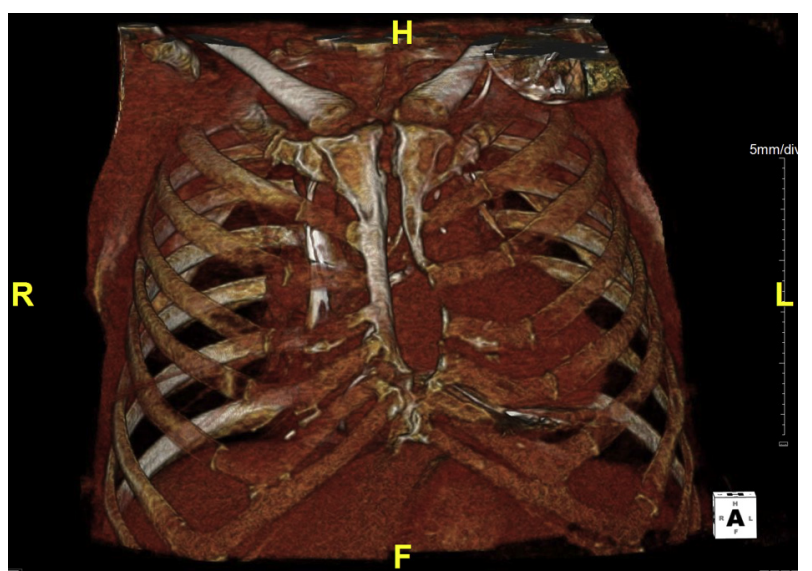

FIGURE 1. The patient's 3-dimensional rendered computed tomography radiograph, which demonstrates marked sternal disruption. The patient had multiple previous breast surgeries, chest wall irradiation for breast cancer, and pectoralis muscle flaps from previous mediastinitis following coronary artery bypass surgery. $H$, Head; $R$, right; $L$, left; $F$, foot.

was $3 \mathrm{~L} / \mathrm{min} \times \mathrm{m}^{2}$ on epinephrine $(4 \mu \mathrm{g} / \mathrm{min})$ and norepinephrine $(9 \mu \mathrm{g} / \mathrm{min})$.

Postoperatively, the patient remained on high-dose inotropes and required increasing vasopressor support. Forty-eight hours following the index operation, the LVAD flow was $1.8 \mathrm{~L} / \mathrm{min}$ on $3200 \mathrm{rpm}$. Left and right arm/femoral artery MAPs were 100 and $60 \mathrm{~mm} \mathrm{Hg}$, respectively. The serum lactate, which initially cleared, increased to $4 \mathrm{mmol} / \mathrm{L}$. To maintain adequate systemic MAP $>65 \mathrm{~mm}$ $\mathrm{Hg}$, the patient required norepinephrine ( $24 \mu \mathrm{g} / \mathrm{min})$, vasopressin $(0.1 \mathrm{U} / \mathrm{min})$, and phenylephrine $(50 \mu \mathrm{g} / \mathrm{min})$. Ultrasound interrogation of the LVAD-axillary artery graft anastomosis demonstrated no hemodynamically significant stenosis. It was surmised that the left axillary artery outflow tract provided insufficient LVAD outflow for corporeal perfusion and ventricular offloading. The ascending and descending aorta were inaccessible due to excessive calcium deposition and dense lung adhesions. To improve the axillary artery outflow and subsequently the flow of the LVAD, an extra anatomic bypass was proposed. The 2 options under consideration included a left axillofemoral bypass and a LARAAB. Due to her morbid obesity and large pannus, it was determined that the LARAAB would be the best configuration to improve the LVAD flow volume while mitigating the risk of femoral dissection and graft infection associated with poor groin incision wound healing.

Through the previous axillary incision, the LVAD graft and native axillary artery distal to the LVAD graft were identified, dissected, and mobilized. A $6 \mathrm{~cm} \times 6 \mathrm{~cm}$ dissection bed hematoma was evacuated. The umbilical tape band was removed. Our initial plan was to bring the graft directly off the LVAD outflow conduit. The LVAD system did not tolerate partial side clamping due to hemodynamic instability. Therefore, we opted to site the anastomosis on the

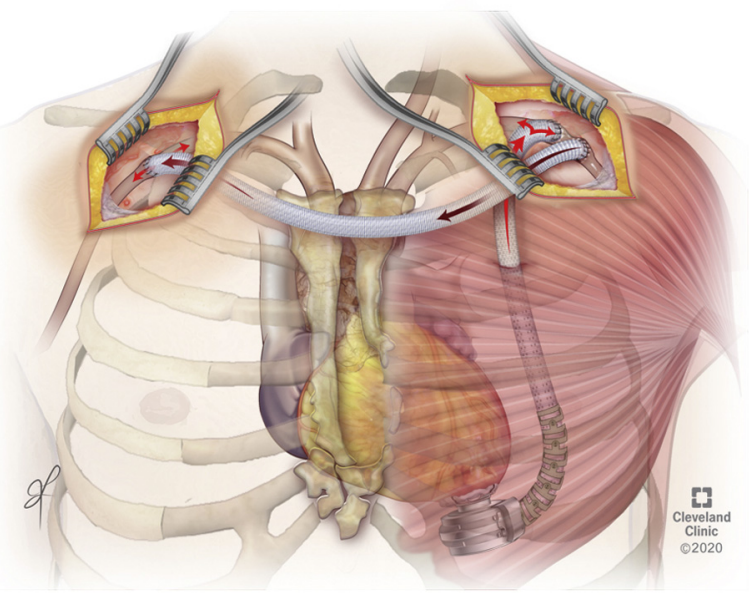

FIGURE 2. A left axillary left ventricular assist device (LVAD) outflow anastomosis followed by left axillary-right axillary arterial bypass (LAR$\mathrm{AAB}$ ) was employed as the alternative site for the LVAD outflow graft in the setting of a porcelain ascending aorta, a hostile chest wall and mediastinum, and a small-caliber axillary artery. A left subcostal incision was employed as a sternotomy-sparing approach. Insufficient LVAD flow was enhanced by creation of LARAAB. The LARAAB represents a potential alternative route to augment LVAD outflow graft in the settings of a heavily calcified ascending aorta and hostile chest wall/mediastinum, with insufficient outflow.

left axillary artery $5 \mathrm{~cm}$ distal to the LVAD outflow graft. The vessel was found to be free of thrombus or dissection. An 8-mm externally supported ringed polytetrafluroethylene graft was tunneled above the pectoralis flap in a left to right configuration (Figure 2). After completing the anastomosis and heparin reversal, there was augmentation in the outflow of the LVAD with flow of $3.0 \mathrm{~L} / \mathrm{min}$ on $3000 \mathrm{rpm}$ with equal MAPs of $80 \mathrm{~mm} \mathrm{Hg}$ in the left/right arms and femoral lines. Replacement of the left axillary artery band was deemed unnecessary.

On postoperative day 5, LVAD flow was $3.8 \mathrm{~L} / \mathrm{min}$ on $3000 \mathrm{rpm}$, without inotropic and vasopressor support. Her vascular reconstruction was interrogated using the serial speed-change echocardiography and duplex ultrasonography of the outflow graft and axillary bypass. The upper extremities had no evidence of hyper/hypoperfusion. Her LVAD flow volumes were stable at $>4 \mathrm{~L}$ throughout the remainder of her hospital stay and the LARAAB remained patent. The patient had several complications during her hospital stay, including a wound infection of the thoracotomy site requiring surgical debridement improved with negative pressure wound therapy, as well as respiratory failure requiring tracheostomy. She was discharged to acute rehabilitation in stable condition with ambulatory status. At 6 months after discharge she has a patent LVAD/LAR$\mathrm{AAB}$ with healed incisions. 


\section{CONCLUSIONS}

To our knowledge, a thorough literature review suggests that this is the first reported description of an extra-anatomic bypass used to facilitate LVAD success. ${ }^{2}$ Proper LV unloading from an LVAD is predicated by arterial resistance and flow in the vessel to which the outflow cannula is anastomosed. Because the outflow graft of the LVAD allows for passive flow of blood, decreasing resistance or afterload of the arterial system facilitates improved LVAD unloading. In this case, the creation of an LARAAB allowed adequate LVAD flows to facilitate proper LV unloading. ${ }^{3}$ A potential area of concern remains the ascending aorta is not receiving washout from LVAD flows; therefore, the patient may be at elevated risk of ascending aortic root thrombus with secondary embolization, myocardial infarction, or coronary artery ostial thrombus. ${ }^{4}$ Nonetheless, the axillary-axillary artery bypass presents a potential novel alternative route for LVAD outflows in the setting of hostile chest wall/mediastinum, inaccessible descending aorta, and insufficient axillary flow due to size.

\section{References}

1. Ahmed MME-S, Aftab M, Singh SK, Mallidi HR, Frazier QH. Left ventricular assist device outflow graft: alternative sites. Ann Cardiothorac Surg. 2014;3: $541-5$.

2. Maltais S, Danter MR, Haglund NA, Schmitto JD, Stulak JM. Nonsternotomy approaches for left ventricular assist device placement. Operat Techn Thorac Cardiovasc Surg. 2014;19:276-91.

3. Doersch KM, Tong CW, Gongora E, Konda S, Sareyyupoglu B. Temporary left ventricular assist device through an axillary access is a promising approach to improve outcomes in refractory cardiogenic shock patients. ASAIO J. 2015;6: 253-8.

4. Kilic A, Acker MA, Atluri P. Dealing with surgical left ventricular assist device complications. J Thorac Dis. 2015;7:2158-64. 